\title{
Evaluation of effect of epidermal growth factor receptor and vascular endothelial growth factor overexpression in survival of patients with glioblastoma multiforme
}

\author{
Babak Alijani', Hamid Saeidi Saedi², Hadi Hajizadeh Fallah ${ }^{3}$, \\ Ehsan Kazemnejad Leili ${ }^{4}$, Adel Kiumarsi ${ }^{1}$, Sasan Andalib ${ }^{5,6,7,8,9,10}$
}

${ }^{1}$ Department of Neurosurgery, Poursina Hospital, School of Medicine, Guilan University of Medical Sciences, Rasht, Iran

2Department of Radiation Oncology, Cancer Research Center, Razi Hospital, School of Medicine, Guilan University of Medical Sciences, Rasht, Iran

3Jam Pathobiology Laboratory, Rasht, Iran

${ }^{4}$ School of Medicine, Guilan University of Medical Sciences, Rasht, Iran

${ }^{5}$ Neuroscience Research Center, Department of Neurosurgery, Poursina Hospital,

School of Medicine, Guilan University of Medical Sciences, Rasht, Iran

${ }^{6}$ Road Trauma Research Center, Poursina Hospital, Guilan University of Medical Sciences, Rasht, Iran

${ }^{7}$ Research Unit of Clinical Physiology and Nuclear Medicine, Department of Clinical Research, Faculty of Health

Sciences, University of Southern Denmark, Odense, Denmark

${ }^{8}$ Department of Nuclear Medicine, Odense University Hospital, Odense, Denmark

${ }^{9}$ Center for Applied Neuroscience, Brain Research - Interdisciplinary Guided Excellence, BRIDGE, Department of

Clinical Research, Faculty of Health Sciences, University of Southern Denmark, Odense, Denmark

${ }^{10}$ Research Unit of Psychiatry, Department of Psychiatry,

Psychiatry in the Region of Southern Denmark, Odense, Denmark

\begin{abstract}
Background. In the central nervous system, glioblastoma multiforme (GBM) is one of the most important neoplasms in adults. Amplification of EGFR and VEGF genes is common in GBM. However, the role of overexpression of EGFR and VEGF in survival of patients with GBM has been a contentious issue. Therefore, we performed the present study to assess the survival rate of patients with EGFR and VEGF overexpression.

Materials and methods. Resected samples of GBM patients were evaluated by immunohistochemistry for overexpression of VEGF and EGFR. The patients were followed up for a year.

Results. The mean \pm sd of age of patients was $56.2 \pm 13.1$ years. The majority of the patients were male (65.9\%). Most of the patients had primary GBM (67\%), notwithstanding 33\% of the patients with secondary GBM. Moreover, $19.2 \%$ and $49.5 \%$ of the samples were VEGF-positive and EGFR-positive, respectively. Overall 6, 12-, and 18 -month survival rates were shown to be $61.5 \pm 5.1 \%, 17.6 \pm 4 \%$, and $4.4 \pm 2.1 \%$, respectively. There was a significant difference between the mean \pm se survival rate in VEGF-negative patients $(12 \pm 1.9$ months) VEGF-positive patients (7.8 \pm 0.5 months) $(\mathrm{P}=0.045)$.

Conclusions. We found an association between VEGF and EGFR over-expression and the survival rate of GBM patients. The VEGF overexpression was significantly associated with the overall survival rate of the GBM patients, but failed to have a prognostic value for their mortality rate. However, EGFR was a predictor of overall survival rate and mortality of GBM patients. Moreover, VEGF overexertion has a stronger association with survival rate in GBM, compared to EGFR overexpression.
\end{abstract}

Keywords: glioblastoma multiforme, EGFR, VEGF, gene overexpression, survival rate 


\section{INTRODUCTION}

GBM, which refers to glioblastoma multiforme, is an important brain tumor in adults. It shows spread invasiveness, angiogenesis, and tumor necrosis. In the central nervous system, malignant gliomas are the most prevalent and lethal cancers of which GBM is the most aggressive. GBM is categorized as WHO grade IV astrocytoma and has poor prognosis (1). The current approved treatment of GBM is surgery, radiotherapy, and chemotherapy with oral temozolomide (2). Patients with malignant glioma have poor survival rate. Prognostic factors such as P53, isocitrate dehydrogenase 1 (IDH1), epidermal growth factor receptor (EGFR), and Ki-67 (3-6) have been established in gliomas.

Substantial research effort has been made to explore genetic changes in GBM. This has led to defining subcategories of GBM with different prognoses and treatment responses (7). Bioinformatics and genome sequencing techniques have helped genome-wide sequence analyses in human cancers (8). The most important genetic alteration in malignant glioma is amplification of EGFR, accounting for about $50 \%$ of all the cases (9). Involved mechanisms are gene amplification and receptor structure alterations due to gene mutation. Common EGFR mutations have been found to be associated with GBM (10).

GBM has angiogenesis and tumorigenesis, in which VEGF (vascular endothelial growth factor) has a role (11). In addition, it serves an important signaling role in the central nervous system $(12,13)$. Upregulation of VEGF in tumors brings about a poor response to treatment and a poor prognosis in glioma patients. Endothelial cells express VEGF. It has been demonstrated that, in GBM, level of VEGF is elevated. This triggers angiogenesis and tumorigenesis of glioblastoma stem cells $(14,15)$. Moreover, VEGF has been demonstrated to be associated with neural stem cell proliferation and it has been shown that glioblastoma stem cells have common features with neural stem cell (16).

We set out this study to evaluate the effect of overexpression of EGFR and VEGF in survival of the patients with GBM.

\section{MATERIALS AND METHODS}

We evaluated patients with GBM during 2013 and 2014 in this retrospective study. The clinical diagnosis had been done by a neurosurgeon. The surgical pathology specimens were obtained from Archives of Department of Surgical Pathology, Poursina Hospital, School of Medicine, Guilan University of Medical Sciences, Rasht, Iran.

\section{Patients}

Post-operative survival duration was obtained from medical records and by phone contact. Crude data included clinical and laboratory data, age at diagnosis, tumor type (primary versus secondary), location of tumor, tumor volume, resected volume, last follow-up or death. Patients with other types of astrocytoma and those who did not continue radiotherapy treatment, were excluded from the study.

\section{Specimen preparation}

All of the surgical specimens after partial or complete resection were fixed in buffered formalin (4\%), embedded in paraffin and kept in an archive. Sections stained with HE (hematoxylin and eosin) were evaluated. Proper blocks were thereafter selected for further analysis. Immunohistochemical assays by anti-EGFR and anti-VEGF antibodies were carried out to determine antigen distributions within the tumors and characterize extent of overexpression. For immunohistochemical staining, sections $(5-\mu \mathrm{m})$ were cut and put on silanized slides. The sections were deparaffinized by a series of xylenes, cleared by means of alcohol, and rehydrated before the assay. At ambient temperature, they were then incubated with primary antibodies of VEGF and EGFR for 20 and $15 \mathrm{~min}$, respectively. All the slides were assessed independently by a pathologist. The stained sections were then evaluated for proliferation index, tumor grading, microvascular proliferation, and necrosis. The present study was approved by ethics committee of Guilan University of Medical Sciences.

Data were analyzed by SPSS (version 19) using Kaplan-Meier tests to estimate overall 18-month survival rate for all patients. The log-rank test was applied in order to compare survival rate between the groups. Moreover, to model predictors, Cox models were applied. Frequency tables and Fisher's exact, Wilcoxon Signed-Rank, and Kruskal-Wallis tests were also applied. A P-value $\leq 0.05$ was considered as statistical significance. Survival rate was shown as mean \pm se. 


\section{RESULTS}

In the present study 116 confirmed GBM patients were initially enrolled; however, 25 subjects were excluded according to exclusion criteria. The mean \pm sd age of the participants was $56.2 \pm 13.1$ years old. $65.9 \%$ of the subjects were male and primary GBM constituted $67 \%$ of the tumors. The tumors were mostly found in the right temporal region $(19.8 \%)$, but least seen in the left occipital region $(1.1 \%)$. Baseline characteristics of the patients with GBM are summarized in Table 1.

TABLE 1. Baseline characteristics of patients with confirmed GBM

\begin{tabular}{|c|c|c|c|}
\hline \multicolumn{2}{|c|}{ Variable } & Frequency & Percent \\
\hline \multirow[t]{3}{*}{ Gender } & Male & 31 & $34.1 \%$ \\
\hline & Female & 60 & $65.9 \%$ \\
\hline & Total & 91 & $100.0 \%$ \\
\hline \multicolumn{2}{|c|}{ Age (Mean $\pm S . D)$} & \multicolumn{2}{|c|}{$56.24 \pm 13.13$} \\
\hline \multirow[t]{3}{*}{ Type } & Primary & 61 & $67.0 \%$ \\
\hline & Secondary & 30 & $33.0 \%$ \\
\hline & Total & 91 & $100.0 \%$ \\
\hline \multirow[t]{9}{*}{ Location } & $\begin{array}{c}\text { Right } \\
\text { Temporal }\end{array}$ & 18 & $19.8 \%$ \\
\hline & $\begin{array}{c}\text { Left } \\
\text { Temporal } \\
\end{array}$ & 13 & $14.3 \%$ \\
\hline & $\begin{array}{l}\text { Right } \\
\text { Frontal }\end{array}$ & 13 & $14.3 \%$ \\
\hline & Left Frontal & 16 & $17.6 \%$ \\
\hline & $\begin{array}{c}\text { Right } \\
\text { Parietal }\end{array}$ & 11 & $12.1 \%$ \\
\hline & $\begin{array}{c}\text { Left } \\
\text { Parietal }\end{array}$ & 16 & $17.6 \%$ \\
\hline & $\begin{array}{c}\text { Right } \\
\text { occipital }\end{array}$ & 3 & $3.3 \%$ \\
\hline & $\begin{array}{c}\text { Left } \\
\text { Occipital }\end{array}$ & 1 & $1.1 \%$ \\
\hline & Total & 91 & $100.0 \%$ \\
\hline \multicolumn{2}{|c|}{ Primary tumor size $(\mathrm{cm} 3)$} & \multicolumn{2}{|c|}{$18.48 \pm 5.47$} \\
\hline \multicolumn{2}{|c|}{ Resected tumor volume $(\mathrm{cm} 3)$} & \multicolumn{2}{|c|}{$15.76 \pm 3.92$} \\
\hline
\end{tabular}

\section{Survival rate analysis}

We found that 83 subjects $(91.2 \%)$ were VEGF-positive and 45 patients of the patients (49.5\%) were EGFR-positive. Kaplan-Meier analysis showed that overall survival rate was $61.56 \pm 5.1$ percent in the 6-months follow up, $17.6 \pm 4.0$ percent after 12 months and $4.4 \pm 2.1$ percent after 18 months (Figure1-a).

\section{VEGF}

One-year and 18-month survival rates (mean $\pm \mathrm{se})$ were $50.0 \pm 17.7 \%$ and $12.5 \pm 11.7 \%$ in VEGF-negative patients, respectively. In VEGF-positive patients, one-year and 18-month survival rates (mean \pm se) were $14.5 \pm 3.9$ and $3.6 \pm 2.0$, respectively. A significant difference was found between mean survival rate of VEGF-negative and VEGF-positive patients (12.0 \pm 1.9 months) $(7.8 \pm 0.5$ months $)$ $(p=0.045)$. The median of survival time in negative-VEGF patients (11 months) was higher than in the positive-VEGF patients ( 8 months) (Figure 1-b).

\section{EGFR}

One-year and 18-month survival rates (mean \pm se) were $21.7 \pm 6.1 \%$ and $21.7 \pm 6.1 \%$ in EGFR-negative patients, respectively. In EGFR-positive patients, one-year and 18-month survival rates (mean \pm se) were $8.7 \pm 4.2$ and $13.3 \pm 5.1$, respectively. There was a significant difference in the mean \pm se survival rate of EGFR-negative $(9.8 \pm 0.74$ months) and EGFR-positive (6.5 \pm 0.58$)$ patients (p $=0.001$ ) (Figure 1-c).

Cox regression analysis showed that VEGF overexpression increased the relative risk of mor-

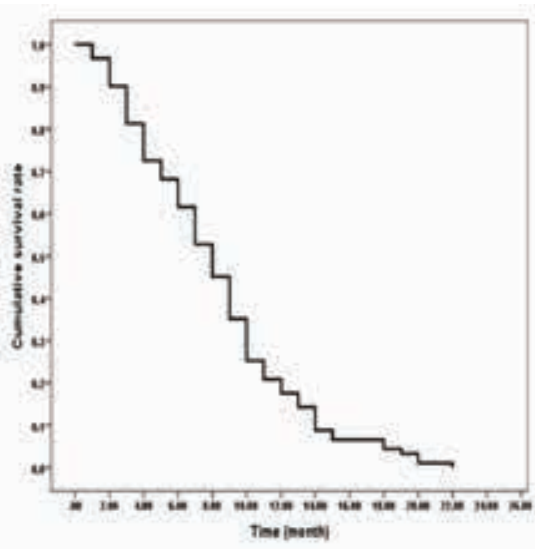

$a$
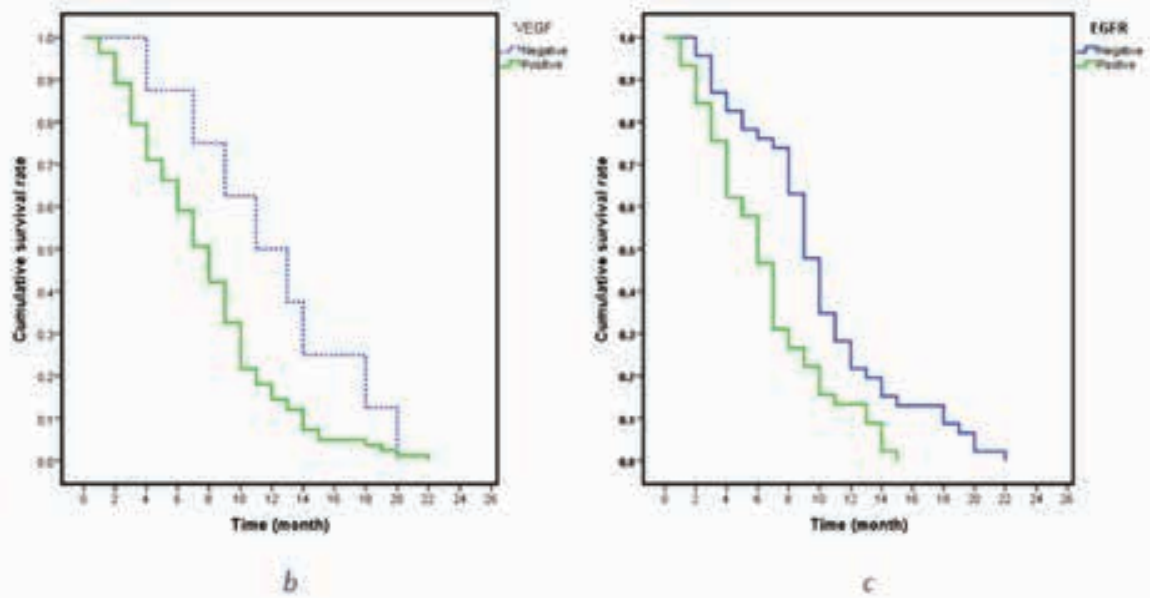

FIGURE 1. Cumulative survival rate of GBM patients: a. total (a); b. by VEGF; c. by EGFR 
TABLE 2. Cox regression of variables affected the mortality of GBM patients

\begin{tabular}{|c|c|c|c|c|c|c|}
\hline Variables & B & SE & Sig. & RR & \multicolumn{2}{|c|}{$95.0 \% \mathrm{Cl}$ for $\operatorname{Exp}(\mathrm{B})$} \\
\cline { 5 - 7 } & & & & & Lower & Upper \\
\hline EGFR & 0.650 & 0.223 & 0.004 & 1.916 & 1.238 & 2.965 \\
\hline Tumor type & -1.034 & 0.238 & 0.0001 & 0.356 & 0.223 & 0.567 \\
\hline
\end{tabular}

tality by 2.26 times $(\mathrm{RR}=2.26,95 \% \mathrm{CI}: 1.08$ 4.74), in contrast to that increased by EGFR, $(2.15$ times) $(\mathrm{RR}=2.15,95 \% \mathrm{CI}: 1.38=3.74)$. Final Cox regression model showed that with matched age, gender and tumor type variables, only EGFR $(\mathrm{RR}=1.9, \mathrm{p}=0.004)$ and tumor type (with trend of secondary to primary tumors) was able to predict the mortality $(\mathrm{RR}=0.36, \mathrm{p}<0.001)$ (Table 2$)$.

\section{DISCUSSION}

In cancers, VEGF is involved in angiogenesis. VEGF also stimulates capillary permeability, angiogenesis and endothelial cell growth (17). Glioma, a highly vascularized tumor, has progressive angiogenesis. Serum and cerebrospinal fluid VEGF has been considered as a potential biomarker in glioma patients $(18,19)$. VEGF upregulation also increases cell density, leading to tumor hypoxia (20). VEGF, which is induced by hypoxia via HIF-1a, forms blood vessels by stimulating VEGFR-2/ $\mathrm{KDR}$ in glioma (21). Additionally, the prognostic value of VEGF upregulation was seen in survival from glioma $(22,23)$. However, it was demonstrated that VEGF failed to be an independent prognostic factor for the survival from glioma patients $(24,25)$. Hence, well-designed studies are still needed to provide evidence for prognostic value of VFGF in glioma (26). EGFR gene amplification and mutations are found in different cancers (27). Therapies have shown limited efficacy against GBM. Use of EGFR-specific inhibitors have been approved for patients with non-small cell lung carcinoma and are currently under investigation by GBM clinical trials $(28,29)$. Specifically, EGF activation of EGFR on glioma cells enhances VEGF secretion by glioma cells (30). In the present study, we evaluated the overexpression of these two growth factors in survival of GBM patients.

We discovered that $91.2 \%$ of patients were VEGF-positive and 49.5\% were EGFR-positive. Both VEGF- and EGFR-positive patients experienced poor 12-month and 18-month survival rate with superiority of VEGF. EGFR and tumor type were two independent prognostic factors of mortality in GBM patients. Chen et al. found higher positive rate of VEGF expression in a group of high-grade glioma, compared to the low-grade glioma (26). It was also reported that VEGF upregulation was involved in the progression of glioma and angiogenesis, suggesting contribution role of VEGF in regulation of neoangiogenesis and proliferation $(31,32)$. We found that VEGF overexpression produced a significantly lower survival rate in GBM patients. Similarly, Cehn et al. (26) in their meta-analysis, implied that VEGF overexpression was associated with overall survival of glioma patients. Subgroup analyses also indicated the strong prognostic value of VEGF overexpression in overall survival of glioma patients. $\mathrm{Xu}$ et al. showed that VEGF heightens proliferation of GBSC through VEGFR2; and Ki8751, which is a VEGFR2-specific inhibitor, makes suppression in VEGF-induced GBSC proliferation (33). Moreover, VEGFR1 impose downregulation in the positive VEGF effect upon the proliferation of GBSCs. Almost all cells, in some GBMs, showed amplification of EGFR, but in others, the tumor cell proportion with amplification of EGFR were 10-60\% in various regions in the same tumor $(34,35)$. EGFR-amplified cells were frequently seen next to the tumor edge, but not in the center (34). However, this was not confirmed by findings of Okada et el. (35). In a case series by Hatanpaa et al. (36), diffuse and focal EGFR gene amplification was seen in $27 \%$ and $9 \%$ of GBMs, respectively. Amongst the GBMs having EGFR overexpression on the membrane, $74 \%$ had diffuse amplification of EGFR gene, and $16 \%$ showed that with focal amplification. Among the GBMs without expression of EGFR on membrane, 0 and $7 \%$ showed diffuse and focal amplification of EGFR gene, respectively.

\section{CONCLUSIONS}

This investigation was the first one carried out in an Iranian population and evaluated the VEGF and EGFR overexpression simultaneously. We 
found an association between VEGF and EGFR over-expression and the survival rate of GBM patients. The VEGF overexpression was significantly associated with overall survival rate of the GBM patients, but failed to have a prognostic value for their mortality. However, EGFR was predictor of overall survival rate and mortality of GBM patients. Moreover, our findings suggest that VEGF overexertion has a stronger association with sur-

Conflict of interest: none declared

Financial support: none declared

\section{REFERENCES}

1. Louis DN, Ohgaki H, Wiestler OD, Cavenee WK, Burger PC, Jouvet A, et al. The 2007 WHO classification of tumours of the central nervous system. Acta Neuropathologica. 2007; 114(2):97-109.

2. Stupp R, Mason WP, Van Den Bent MJ, Weller M, Fisher B, Taphoorn MJ, et al. Radiotherapy plus concomitant and adjuvant temozolomide for glioblastoma. N Engl J Med. 2005;352(10):987-96.

3. Zou P, Xu H, Chen P, Yan Q, Zhao L, Zhao P, et al. IDH1/IDH2 mutations define the prognosis and molecular profiles of patients with gliomas: a meta-analysis. PloS One. 2013;8(7):e68782.

4. Huncharek M, Kupelnick B. Epidermal growth factor receptor gene amplification as a prognostic marker in glioblastoma multiforme: results of a meta-analysis. Oncology Research Featuring Preclinical and Clinical Cancer Therapeutics. 2001;12(2):107-12.

5. Levidou G, El-Habr E, Saetta AA, Bamias C, Katsougiannis K, Patsouris E, et al. P53 immunoexpression as a prognostic marker for human astrocytomas: a meta-analysis and review of the literature. Journal of Neuro-oncology. 2010;100(3):363-71.

6. Chen W-J, He D-S, Tang R-X, Ren F-H, Chen G. Ki-67 is a valuable prognostic factor in gliomas: evidence from a systematic review and meta-analysis. Asian Pac J Cancer Prev. 2015;16(2):411-20.

7. Mellinghoff IK, Wang MY, Vivanco I, Haas-Kogan DA, Zhu S, Dia $E Q$, et al. Molecular determinants of the response of glioblastomas to EGFR kinase inhibitors. N Engl J Med. 2005;2005(353):2012-24.

8. Sjöblom T, Jones S, Wood LD, Parsons DW, Lin J, Barber TD, et al. The consensus coding sequences of human breast and colorectal cancers. Science. 2006;314(5797):268-74.

9. Furnari FB, Fenton T, Bachoo RM, Mukasa A, Stommel JM, Stegh A, et al. Malignant astrocytic glioma: genetics, biology, and paths to treatment. Genes \& Development. 2007;21(21):2683-710.

10. Gan HK, Kaye AH, Luwor RB. The EGFR vlll variant in glioblastoma multiforme. Journal of Clinical Neuroscience. 2009;16(6):748-54.

11. Greenberg DA, Jin K. From angiogenesis to neuropathology. Nature. 2005;438(7070):954.

12. Mackenzie F, Ruhrberg $C$. Diverse roles for VEGF-A in the nervous system. Development. 2012;139(8):1371-80.

13. Rosenstein JM, Krum JM. New roles for VEGF in nervous tissue beyond blood vessels. Experimental Neurology. 2004; 187(2):246-53.

14. Folkins C, Shaked Y, Man S, Tang T, Lee CR, Zhu Z, et al. Glioma tumor stem-like cells promote tumor angiogenesis and vasculogenesis via vascular endothelial growth factor and stromal-derived factor 1. Cancer Research. 2009;69(18):7243-51.

15. Mentlein R, Forstreuter F, Mehdorn HM, Held-Feindt J. Functional significance of vascular endothelial growth factor receptor expression on human glioma cells. Journal of Neuro-oncology. 2004;67(1):9-18.

16. Xiao Z, Kong Y, Yang S, Li M, Wen J, Li L. Upregulation of Flk-1 by bFGF via the ERK pathway is essential for VEGF-mediated promotion of neural stem cell proliferation. Cell Research. 2007;17(1):73. vival rate in GBM, compared to EGFR overexpression. Further investigation is required to better understand the role of VEGF and EGFR overexpression in GBM.

\section{Acknowledgement}

We thank Guilan University of Medical Sciences for its support.

17. Hoeben A, Landuyt B, Highley MS, Wildiers H, Van Oosterom AT, De Bruijn EA. Vascular endothelial growth factor and angiogenesis. Pharmacological Reviews. 2004;56(4):549-80.

18. Peles E, Lidar Z, Simon AJ, Grossman R, Nass D, Ram Z. Angiogenic factors in the cerebrospinal fluid of patients with astrocytic brain tumors. Neurosurgery. 2004;55(3):562-8.

19. Sampath $P$, Weaver CE, Sungarian A, Cortez S, Alderson L, Stopa EG. Cerebrospinal fluid (vascular endothelial growth factor) and serologic (recoverin) tumor markers for malignant glioma. Cancer Control. 2004;11(3):174-80.

20. Rijken PF, Bernsen HJ, Peters JP, Hodgkiss RJ, Raleigh JA, van der Kogel AJ. Spatial relationship between hypoxia and the (perfused) vascular network in a human glioma xenograft: a quantitative multi-parameter analysis. Int J Radiat Oncol Biol Phys. 2000 Sep 1;48(2):571-82.

21. Kaur B, Khwaja FW, Severson EA, Matheny SL, Brat DJ, Van Meir EG. Hypoxia and the hypoxia-inducible-factor pathway in glioma growth and angiogenesis. Neuro-oncology. 2005;7(2):134-53.

22. Chan AS, Leung SY, Wong MP, Yuen ST, Cheung N, Fan YW, Chung LP. Expression of vascular endothelial growth factor and its receptors in the anaplastic progression of astrocytoma, oligodendroglioma, and ependymoma. Am J Surg Pathol. $1998 \mathrm{Jul}$; 22(7):816-26.

23. Abdulrauf SI, Edvardsen K, Ho KL, Yang XY, Rock JP, Rosenblum $\mathrm{ML}$. Vascular endothelial growth factor expression and vascular density as prognostic markers of survival in patients with low-grade astrocytoma. Journal of Neurosurgery. 1998;88(3):513-20.

24. Korshunov A, Golanov A. The prognostic significance of vascular endothelial growth factor (VEGF C-1) immunoexpression in oligodendroglioma. An analysis of 91 cases. Journal of Neuro-oncology. 2000;48(1):13-9.

25. Korshunov A, Golanov A, Timirgaz V. Immunohistochemical markers for prognosis of ependymal neoplasms. Journal of Neuro-oncology. 2002;58(3):255-70.

26. Chen W, He D, Li Z, Zhang X, Pan D, Chen G. Overexpression of vascular endothelial growth factor indicates poor outcomes of glioma: a systematic review and meta-analysis. Int J Clin Exp Med. 2015 Jun 15;8(6):8709-19.

27. Nagane M, Lin H, Cavenee WK, Huang H-JS. Aberrant receptor signaling in human malignant gliomas: mechanisms and therapeutic implications. Cancer Letters. 2001;162:S17-S21.

28. Raizer JJ, Abrey LE, Lassman AB, Chang SM, Lamborn KR, Kuhn $J G$, et al. A phase II trial of erlotinib in patients with recurrent malignant gliomas and nonprogressive glioblastoma multiforme postradiation therapy. Neuro-oncology. 2009;12(1):95-103.

29. Ji H, Sharpless NE, Wong K-K. EGFR targeted therapy: view from biological standpoint. Cell Cycle. 2006;5(18):2072-6.

30. Goldman CK, Kim J, Wong WL, King V, Brock T, Gillespie GY. Epidermal growth factor stimulates vascular endothelial growth factor production by human malignant glioma cells: a model of 
glioblastoma multiforme pathophysiology. Mol Biol Cell. 1993 Jan;4(1):121-33.

31. Hlobilkova A, Ehrmann J, Knizetova P, Krejci V, Kalita O, Kolar Z. Analysis of VEGF, Flt-1, Flk-1, nestin and MMP-9 in relation to astrocytoma pathogenesis and progression. Neoplasma. 2009;56(4):284.

32. Ma C, Li Y, Zhang X, Zhao G, Xu H. Levels of vascular endothelial growth factor and matrix metalloproteinase- 9 proteins in patients with glioma. Journal of International Medical Research. 2014;42(1):198-204.

33. Xu C, Wu X, Zhu J. VEGF Promotes Proliferation of Human Glioblastoma multiforme Stem-Like Cells through VEGF Receptor 2. The Scientific World Journal. 2013;2013:8.
34. Kleinschmidt-DeMasters BK, Lillehei KO, Varella-Garcia M. Glioblastomas in the older old. Arch Pathol Lab Med. 2005 May;129(5):624-31.

35. Okada Y, Hurwitz EE, Esposito JM, Brower MA, Nutt CL, Louis DN. Selection pressures of TP53 mutation and microenvironmental location influence epidermal growth factor receptor gene amplification in human glioblastomas. Cancer Research. 2003;63(2):413-6.

36. Hatanpaa KJ, Burma S, Zhao D, Habib AA. Epidermal Growth Factor Receptor in Glioma: Signal Transduction, Neuropathology, Imaging, and Radioresistance. Neoplasia (New York). 2010;12(9):675-84. 